\title{
On the development of Agent-Based Models for infrastructure evolution
}

\section{Igor Nikolic* and Gerard P.J. Dijkema}

Both authors are affiliated with Section Energy and Industry, Faculty of Technology Policy and Management, Delft University of Technology, Jaffalaan 5, 2628 BX, Delft, The Netherlands Fax: +31152783422 E-mail: i.nikolic@tudelft.nl

*Corresponding author

\begin{abstract}
Infrastructure systems for energy, water, transport, information etc. are large scale socio-technical systems that are critical for achieving a sustainable world. They were not created at the current global scale at once, but have slowly evolved from simple local systems, through many social and technical decisions. If we are to understand them and manage them sustainably, we need to capture their full diversity and adaptivity in models that respect Ashby's law of requisite variety. Models of evolving complex systems must themselves be evolving complex systems that can not be created from scratch but must be grown from simple to complex. This paper presents a sociotechnical evolutionary modeling process for creating evolving, complex agent based models for understanding the evolution of large scale sociotechnical systems such as infrastructures. It involves the continuous co-evolution and improvement of a social process for model specification, the technical design of a modular simulation engine, the encoding of formalized knowledge and collection of relevant facts. In the paper we introduce the process design, the requirements for guiding the evolution of the modeling process and illustrate the process for Agent Based Model development by showing a series of ever more complex models.
\end{abstract}

Keywords: agent based modeling, evolution, evolutionary process design, infrastructure evolution

Reference to this paper should be made as follows: Igor Nikolic and Gerard P.J. Dijkema (xxxx) 'On the development of Agent-Based Models for infrastructure evolution', Int. J. of Critical Infrastructures, Vol. $\mathrm{x}$, No. $\mathrm{x}, \mathrm{pp} \cdot \mathrm{xxx}-\mathrm{xxx}$.

Biographical notes: ir. Igor Nikolic is an Assistant Professor, specializing in applying complex adaptive systems theory, agent based modeling and evolutionary theory to model industryinfrastructure network evolution

Dr. ir. Gerard P.J. Dijkema is a Associate Professor with expertise covering networked process system innovation, systems dynamics modeling of large-scale systems and the relation between industrial-infra systems and applicable policy, law and economics 


\section{Introduction}

\subsection{Evolving world}

The global industrial system and the infrastructure systems that support it form the backbone of modern human society. Infrastructures are large-scale systems spanning nations, continents if not the globe. Their lifetime is measured in decades up to centuries and society allocates large amounts of resources to their construction and maintenance. Their development is strongly path dependent, while at the same time they strongly shape the future direction of the society that depends on them. They provide the basic structure that is instrumental in our production and consumption patterns, and are therefore directly responsible to the overall sustainability of the human race.

In order to structure our thinking on infrastructure systems, we consider them to be Large Scale Socio-Technical Systems [Bijker et al., 1987], a class of systems that span technical artifacts embedded in a social network. In order to avoid the lengthy acronym, we will use the term $\lambda$-systems[Nikolic et al., 2008] throughout this work. $\lambda$-systemsinclude social elements such as operating companies, investors, local and national governments, regional development agencies, non-governmental organizations, customers and institutions. These develop around, sustain and depend on particular technical systems, be it a single plant, industrial complex or set of interconnected supply-chains. Examples of $\lambda$-systems are regional industrial clusters, power grids, multimodal transport networks and telecommunication networks.

While the technical components of $\lambda$-systems can be engineered in relative isolation once their design requirements have been set, their social networks and the emergent system structures currently cannot. Instead, their state and structure we observe today have evolved as the result of a series of discrete decisions by a myriad of involved stakeholders, who act to pursue or safeguard their interests. Each actor is subject to influence, pressure, opportunities and constraints from within and external to the network. Global markets, national and international rules and regulations, availability of and access to capital, knowledge and skilled labor are external to $\lambda$-systems. Internal pressures are caused by, e.g., changes in the composition and preferences of the working populations, or by replacement of old assets with new, more advanced ones. Thus, the global industrial system, our industrial society, can be viewed as a collection of co-evolved $\lambda$-systems internal structures and functions change over time.

In the traditional scientific paradigm, since Bacon, to increase our understanding one would break a system down into small components to be tested and experimented on in isolation.

Consider, for example, the question what the effect of carbon emission-trading would be on electric power production [Chappin et al., 2009]. To answer this question among others requires knowledge on available technology, power system design and economics but also insight into the strategy and decision-making of elec- 
tric power companies. To elucidate the latter, we may study single electric power producing company strategy by investigating their response the introduction of emission-trading. However, even if we include in this investigation how companies observe and react to other companies, we still cannot give an underpinned answer to the original question, because the electric power production sector is an $\lambda$-systems, where the configuration and characteristics of technical and social elements determine the interaction taking place withing the system, the perception and response to external market signals and trends. To extract the system's response to emissiontrading using a reductionist approach is impossible. To only begin doing this and contribute to the sustainable management of this and other $\lambda$-systems we need a holistic paradigm.

In complex system theory, the central principle is that all phenomena can be described by interconnected networks of simple units. This approach, also called Generative Science [Epstein, 1999] describes complex behaviors as a generative process. In this approach, deterministic, finite rules and parameters interact to generate indeterministic and infinite system behavior. In generative science, the agent or individual component, is the theory, as it is often said. By having an explicit behavioral model of an individual, or the smallest level component, we can build/generate explanations of the observed phenomena.

Indeed, according to Mikulecky [2001] complex systems like $\lambda$-systems can only be understood when studied from multiple perspectives requiring multiple formalisms. That is, integrating knowledge of various domain and disciplinary experts in a formalized way is required for adequate and sufficiently complete capturing of the properties and behavior of a complex system. Furthermore, studying $\lambda$-system from the traditional reductionist paradigm a priori usually results in models that assume static components interaction structure, removing from view the emergent and evolutionary properties of $\lambda$-systems, while this is exactly the behavior that is of interest to researchers and planners in $\lambda$-systems.

Let an agent represent some individual, relatively autonomous entity such as a cell, a species, an individual, a firm, a nation. Complex Adaptive Systems (CAS) can then be defined as a "dynamic network of many agents acting in parallel, constantly acting and reacting to what the other agents are doing. The control of a CAS tends to be highly dispersed and decentralized. If there is to be any coherent behavior in the system, it has to arise from competition and cooperation among the agents. The overall behavior of the system is the result of a huge number of decisions made every moment by many individual agents." [Waldorp, 1992]

\subsection{Problem formulated}

The development of industrial and infrastructure system is evolutionary, and thus intractable [Dennet, 1996], meaning that its exact future development can never be fully predicted. Only way to reliably predict the future state of an evolving system is to simply perform an action and observe its effects over time. Real world experiments are clearly impossible.

Our hypothesis is that the use of adequate models of $\lambda$-systems evolution, while unable to exactly predict the future, will improve decision-making on infrastructure systems by examining possible evolutionary pathways. Using appropriate models we can reduce uncertainty by examining possible future states and communicate 
the insights back to the systems stakeholders.

The main question answered in this paper is how do we go about creating evolving, complex models of large scale socio-technical systems, and what are the requirements for such modeling process?

Ashby's Law of Requisite Variety [Ashby, 1968] states: "The variety in the control system must be equal to or larger than the variety of the perturbations in order to achieve control." Paraphrasing, a model system or controller can only model or control something to the extent that the model has sufficient internal variety to represent the real system. This implies that if we are to build successful models of complex $\lambda$-systems, the models themselves need to be $\lambda$-systems. The modelling tool for describing a $\lambda$-system, must therefore be able to contain multi-domain and multi-disciplinary knowledge, be generative and adaptive and consist of a social and technical component. That is, it must exhibit adaptive, emergent behavior through interactions of adaptive heterogeneous low-level elements. Furthermore, Gall [2002] provides us with a important observation on complex systems: "A complex system that works is invariably found to have evolved from a simple system that worked. The inverse proposition also appears to be true: A complex system designed from scratch never works and cannot be made to work. You have to start over, beginning with a working simple system."

Paraphrasing Gall, if the result of a modeling process is to be a successful evolving model of a Complex Adaptive System, the process of creating it must also be complex and adaptive. We are not only making generative models; we also need to have a generative process for creating these models. This insight leads us to the central paradigm of the modelling process presented in this paper:

If we are to model a $\lambda$-systems evolution, not only must our models be Complex Adaptive Systemss, but the process for creating those models must itself be a complex, evolving process. We must start with a simple process for generating simple models, and evolve so that over time a complex process will generate complex models.

This paper focuses on describing the design of a evolutionary and adaptive process for creating models of evolving, complex system.

\section{Evolutionary modeling process}

In order to model the co-evolution of a socio-technical system, a co-evolutionary socio-technical approach must be used. Our approach consists of an evolving series of models, through case studies detailing and continually improving the social knowledge collection process, elaborating the simulation engine, collecting new multiformal knowledge and collecting new facts. This process is continually generating hypotheses for future work and conclusions in both social process and technical design development. Allowing for the evolutionary nature means acknowledging the path dependent, adaptive, chaotic and intractable nature of the process we are involved in.

The process of creating ever more successful models is conceptualized as an evolutionary process involving four aspects of the model creation process. They are:

Technology Technical aspects of model building: which software and hardware 
system to use, how to organize the modelling software components, how to store data, how to analyze results, how to scale the model, etc.

Social process The design of the social process of involving stakeholders in identifying and collecting relevant knowledge and providing feedback on the model's outcomes. Relevant aspects include how to select the right participants, the script of the collaborative process, the manner in which feedback is organized, etc.

Domain Knowledge The formalized and encoded domain knowledge representation of $\lambda$-systems. Microeconomics, chemical engineering, psychology, etc. are examples of formalized domain knowledge.

Facts The factual information that describes components of the $\lambda$-system, their interactions and overall system behavior. Examples are specific processing plants, their in- and outputs, economic performance data, etc.

The basic assumption of the co-evolutionary process is that it proceeds in steps or generations consisting of case studies. Each case generates insights that are used to improve the modelling process of the following case. We can envision the four aspects being locked in a coupled fitness landscape [Hordijk and Kauffman, 2005], shaping each others fitness and influencing each others development over time, just as, for example, zebras grazing on the savanna shape the evolution of the grass they graze on, and the lions shape the evolution of the zebras, and indirectly of the grass as well

In order to ensure knowledge generation, the modelling process leading to each case is unbalanced, that is, one aspect will be extended/explored more than the others. For example, a novel modelling tool will be tested, while keeping the social and factual designs constant, in order to learn about its effectiveness. The goal is to keep a ceteris paribus assumption as much as possible, as is usual in modelling work. We are of course limited by the practical requirements of the case study, so this might not always be possible. This process of unbalanced process development is illustrated in Figure 1.

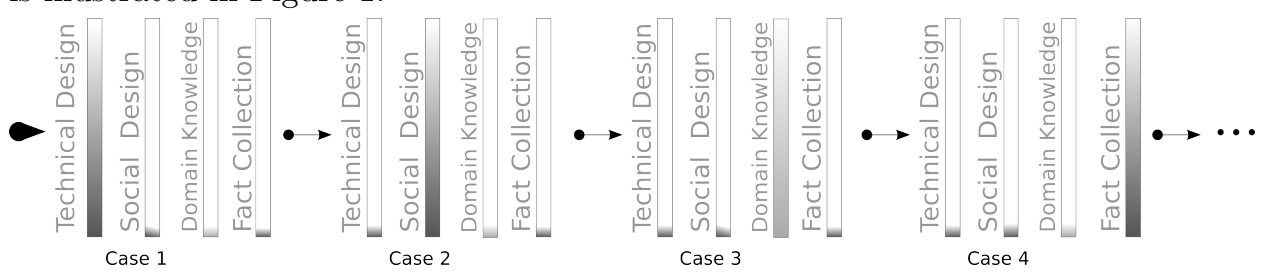

Figure 1 Initial process steps

In Figure 1 each block represents a case study with a corresponding model. The colored bars depict the height of the deformation of the coupled fitness landscape in that dimension. Each case starts with a number of hypotheses. These hypotheses are at two levels:

- Whether the planned extension of the methodology will prove to be useful; and

- Whether the specific case assumptions will hold true. 
We assume that we start with the following technical feasibility hypothesis: It is possible to technically design a model that has the necessary properties to model $\lambda$-system evolution. If this hypothesis is not rejected, we will proceed to design a social process. The next hypothesis is: The proposed social process design is able to involve stakeholders in modelling and understanding $\lambda$-system evolution. The third step starts with the hypothesis: The collected and formalized domain knowledge about the $\lambda$-system under study is useful in useful in understanding the system's evolution. The fourth hypothesis is: The collected facts on the $\lambda$-system under study will allow for useful model creation and novel insights into the system's evolution process. After these four initial steps, the modelling process is extended in whichever direction offers greatest insights, and the direction it will take cannot be predicted in advance.

It may be seen there exists no end to this process. While theoretically grounded stop criteria might be developed, in a more practical sense, one stops when the project defined with the stakeholders is completed and the stakeholders are satisfied and/or the resources for modelling are consumed.

Evolution is only possible if each aspect continually deforms the fitness landscape [Kauffman and Johnsen, 1991]. In this case this means that each aspect contributes new questions and insights to the model development process, making itself more visible or fit by providing insights and results and by exposing the inadequacies of other aspects. In order to illustrate the co-evolution between these four aspects making up a modelling process, a simplified example is presented below.

In this example, a Regional Development Agency (RDA) asks a process engineer to create a model of a local industrial network with associated infrastructure, so that better decisions can be made about which type of company to invite to join the cluster. A co-evolutionary modelling process could look like this :

Generation 1 The engineer creates a simple model consisting of agents that have mass in- and outflows. It is built by one person and is based on process engineering knowledge. It encodes facts about the relevant mass flows and it accurately represents the mass flow structure in the region. However, the RDA would like to know how the cluster could react to a change of the oil price.

Generation 2 An MBA is invited into the team, and the model's technical design is extended to encode product pricing, company assets and price setting logic. This model now encodes both process engineering and corporate finance knowledge. The model shows possible network structures, depending on oil price levels. The response, however, is unrealistic, as there is no knowledge included yet on global oil price dynamics.

Generation 3 A macroeconomist is involved. She provides knowledge on longterm global price movements, and observes that the capital market interest rate changes over time as well. This prompts a change in the model design to enable agents to borrow money in order to invest in new facilities. The model is run and provides possible cluster development pathways, depending on different oil price and interest rate scenarios.

Generation 4 A resident psychologist realizes that firms risk attitudes will be relevant if they are to fit in the cluster. The model design is changed, adding the ability to encode risk perception, and since this considerably increases the parameter dimensionality, the model scheduler is adapted to allow model execution on the High Performance Computing cluster. As the outcomes are evaluated against 
possible agent risk strategies, the RDA estimates that they need to invite a bioelectricity producer with high risk tolerance to join their cluster in order to allow it to grow. However, it is realized that there is a lack of precise factual information on bioelectrical processes, so a data collection campaign is begun. The process is continued until the RDA is satisfied.

\section{Steering the evolutionary modeling process}

The modeling process described above is evolutionary. Evolution requires some form of selection to be present in order to function. How can we know which improvement to keep and which one to remove? Changes between two generations are improvements only if they are 'fit', that is, if they provide us with added useful knowledge or generate new questions. If the fitness is low, the change should not be used. Fitness is not only defined relatively between the species, but also relative to their environment. In this case, the co-evolutionary process between species takes place in a dynamic, socially constructed environment shared by those species. The environment of this co-evolutionary process consists of the modelers and the stakeholders who determine what is useful and what is not. This social environment contains elements that are external to the four interacting species, such as the sudden availability of resources (money, data, case studies) forcing the co-evolutionary process into an unexpected direction. This process also works in reverse. The judgment of usefulness by the stakeholder is determined by current and past performance of the species. The co-evolutionary process also shapes the expectations of the stakeholders, inspiring them to try new things or discouraging other courses of action. During this process, the environment of the co-evolutionary process adapts, and in essence co-evolves with the four aspects.

In this way, a constant deformation of the fitness landscape, i.e., a constant push to change and improve, is created. Constant hypothesis creation and falsification becomes possible. For example, improve the technical part, see what that teaches you; keep the best technical insights and improve the social part, and see what new knowledge it provides you, etc. Every new evolutionary move/hypothesis generates new knowledge. The process mimics the natural processes of variation and multiplication [Darwin, 1985]. Multiple new ideas are created from older ones, effectively creating knowledge generations.

This modeling process, being co-evolutionary is chaotic, sensitive to small perturbations that can have large effects over time. In order to guide its evolution in a robust manner, guiding principles and requirements for the process need to be defined.

\subsection{Guiding principles}

First we will present the guiding principles of the modeling process and use them to create the requirements for the co-evolutionary modeling process. Given the evolutionary nature of this problem, there can be no guarantee that the list of principles is complete. Other sets may be possible. However, we conjecture that the presented principles are sufficient for defining a useful set of requirements for the process. 
Requisite variety The modeling process must have similar properties to the system it is trying to model. It has to be isomorphic (many discrete entities interacting), isodynamic (components and overall system evolve over time), span multiple formalisms (e.g. engineering, law, psychology) and respect the social and technical reality (not break any laws of nature and be socially realistic).

Local optimization Evolution, given its algorithmic nature does not produce the "best possible" solution. In other words, evolution is a local, not a global optimizer, creating solutions that are good enough for the given situation [Dennet, 1996, Darwin, 1985]. Thus, when we consider being in a evolutionary process, the notion of "good enough" is important. There are two main reasons for this. First, perfect design is impossible to determine, since the environment (and thus the fitness landscape) is constantly changing. Second, even if we could determine the perfect design, it would certainly be a waste of resources, since good enough is exactly that.

No termination criterion Evolutionary processes do not have a termination criterion. That is, one can not determine when to stop. Natural systems do not progress, in a sense of a gradual improvement process, but instead simply proceed or advance as time.

Path dependency Natural evolution is sometimes summarized as more and more variation on less and less themes. It allures to the very strongly path dependent nature of evolution. Evolution modifies and adapts the material it has at hand, only rarely coming up with totally new designs [Dawkins, 1990].

Shared standards Natural systems have a several layers of universal interface between species. At the basic level, the DNA coding is universal. DNA codons ( the base triplet encoding for a certain amino acid) is universal to all life. In the technical domain, intercontinental transport is standardized in containers, and data flows are standardized in the TCP/IP protocol.

Shared effort Any evolutionary process is necessarily a co-evolutionary process; one does not evolve alone. Furthermore, when we consider the notion of multiple formalisms as discussed above it becomes obvious that a shared, concerted action is necessary to tackle a multi-formal problem. Furthermore, newest insights in the importance of social diversity in problem solving processes [Page, 2007] reinforce this idea.

\subsection{Requirements}

Given the previous discussion on guiding principles, it is possible to define requirements for the evolutionary process of framework development. Herder [1999] defines functional requirements as specifications of functions that the design must provide. We differentiate functional requirements at two levels: process and outcome. Process requirements help shape the modeling process design. They are specific and based on the guiding principles of evolution and modelling. The outcome requirements help establish whether the models created by the modelling process are good enough. They are fairly generic and are based on the usual criteria for scientific output. A summary of the requirements is presented in Table 1 .

The requirements presented below are used by the stakeholders guiding the evolutionary process as a set of questions that can be asked to check whether 
Table 1 Overview of Process and Outcome Requirements

\begin{tabular}{|l|l|}
\hline Process & Outcome \\
\hline \hline Open Source & Useful \\
Sufficient community diversity & Testable \\
Organically growing & \\
Unchangeable history & \\
Enforceable authorship & \\
Modular & \\
\hline
\end{tabular}

the evolving modeling process is still on track. When an change in one of the four aspects creates a situation in which the answer to the requirements becomes negative, that change must be dropped.

\subsection{Process requirements}

Open Source Scientific work has traditionally been based on peer review. Upon completion of the work, the research process, data and results are presented for inspection, repetition, confirmation and improvement to others. However, much of the work is still done behind closed doors, and only when deemed ready is it carefully released. While this has worked well, new multidisciplinary problems require more radical forms of collaboration [Kepler et al., 2006].

We believe that radical collaboration can only be ensured by keeping the work fully open source during development. Furthermore, when the computer code used and developed is open source, it encourages standardization. This is captured in the guiding principle of shared standards. Why reinvent the wheel, when a perfectly functional implementation is freely available? Open source computer code essentially is a freely accessible shared knowledge base that promotes community building, since it is possible for others to get involved. Thus, work developed and published with open access in mind has a greater research impact than when it is not freely available [Antelman, 2004]. Therefore, this requirement can be summed up as: Is the work openly accessible?

Sufficiently diverse community This requirement is based in the guiding principle of requisite variety [Ashby, 1968] and shared effort. As already discussed before $\lambda$-system can never be understood by a single formalism or a single person. In order to successfully undertake a large and complex process of understanding $\lambda$-system a creation of a diverse social network is necessary [Page, 2007]. Diversity of people involved in the process has to be large enough to cover all the main conceptual areas needed in the modeling effort. Therefore, this requirement can be summed up as: Is there a sufficient number of others involved?

Organically growing Based on the guiding principle of local optimization the requirement of organic growth can be formulated. Each individual of the species "wants" just one thing; to survive and reproduce [Darwin, 1985, Dawkins, 1990]. This individually selfish behavior creates a overall better species. In social systems mutually contingent self interest serves a similar purpose [Karp, 2006]. All participants is a social system work on improving their own well being, wealth or status 
by collaborating on a creation of something much larger than they could create alone without limiting the other. Systems that consist of elements that have such internal motivation can be seen to grow organically from the inside out. Therefore, this requirement can be summed up as: Does it grow from the inside out?

Unchangeable history Based on the principle of path dependence and in order to fulfill the requirement of falsifiability, the methodology and the work done according to it must have as accurate and immutable historic record as possible [Westerberg et al., 1997]. Achieving a historic record is traditionally done by keeping a revision history of formalized knowledge. For example, the project specifications document has all of its previous versions available for inspection. Full versioning basically means that we can go back to any point in time during the development of the process or the model and be able to recover the state of knowledge and factual information that was available at that time. If the development process finds itself in a dead end, we must be able to go back to those decision points undo/redo them. Therefore, this requirement can be summed up as: Is the historic record accessible?

Enforceable authorship This requirement is based on the principle of local optimization and shared effort. Enforceable authorship is a requirement to know "who did what and when". In a well designed system, this requirement is automatically achieved in concert with the previous one. There are two reasons for this requirement. First, since we are involved in a social process of methodology development and model building, no matter how good our record keeping is, there will always be aspects of design decisions and fact collection that are not recorded. Record of authorship provides a link to the social memory of the group. Second reason is that in a academic environment it is important to give credit where credit is due. Therefore, this requirement can be summed up as: Is it recorded who did what and when?

Modular The functional requirement of modularity is based on the guiding principle of shared standards. The design must be such that any component can be replaced without breaking the overall functionality [Egyedi and Verwater-Lukszo, 2005]. The replacement can be either functionally identical but implemented differently, or the replacement could be extending the functionality. In a sense, the entire design can be seen as a System Of Systems design [DeLaurentis and Crossley, 2005], where the overall system consists of coupled components, the components have porous boundaries and have a meaningful functionality by themselves. Furthermore, modularity also implies re usability. By creating a consistently modular design, we are effectively building up a library of components that can be recombined at will to create novel models or methods. Therefore, this requirement can be summed up as: Does it consist of interchangeable pieces?

\subsection{Outcome requirements}

Based on the guiding principles and the process requirements discussed above, we can extract a number of functional specifications that the modeling process outcome must fulfill.

Useful The usefulness requirement is based on the guiding principle of local optimization. Nature does not make things what will be useful one day. Only ones that are useful now. We need to specify to whom it is useful. Two answers are possible. Useful to the stakeholder involved in the $\lambda$-system modeling, or useful to 
the modeler designing the modeling process. Given this, usefulness is a somewhat ambiguous requirements, since it can easily be made always true. Even when a step in the process or a case fails to produce wanted results, we can still learn what does not work, and is thus useful. Usefulness is not an objective criterion. Usefulness can only be determined in interacting between the stakeholders and the modelers. In order to determine it a social process is needed. This requirement can be summed up as: Did it deliver useful insights to stakeholders or modelers?

Testable The most important requirement for any knowledge generated within the scientific endeavor is falsifiability or testability [Popper, 2002]. First, as specified by the open-source requirement, the computer code and data that the model consists on must be known. Second, each model run must be fully repeatable. In model that has stochastic components two further requirements are necessary in order to ensure repeatability. First, unchangeable history allows for each developed model to be retrieved and rerun. Second, by using a open source deterministic pseudorandom generator and a known seed value, all the (pseudo)stochastic elements of the models can be exactly repeated. Model that meets the above specifications is fully repeatable and thus testable. Please note that this only concerns that verification of a model, not its validation. That is handled by the usefulness requirement. This requirement can be summed up as: Can it be tested?

\section{Models evolved}

Several case studies have been developed using the evolving system designed in the guiding principles and requirements [?]. As will be shown, the use of a common platform creates an environment where people focusing on different problems can leverage others diverse approaches and insights into further improvements in the modeling platform. As showed below, the setup allows for a broad variety of models. This strategy means that others efforts are not lost but contribute to a design process involving the iterative refinement of collective knowledge. Figure 2 describes the genealogical tree of the models developed using the co-evolutionary modeling process presented in this paper.

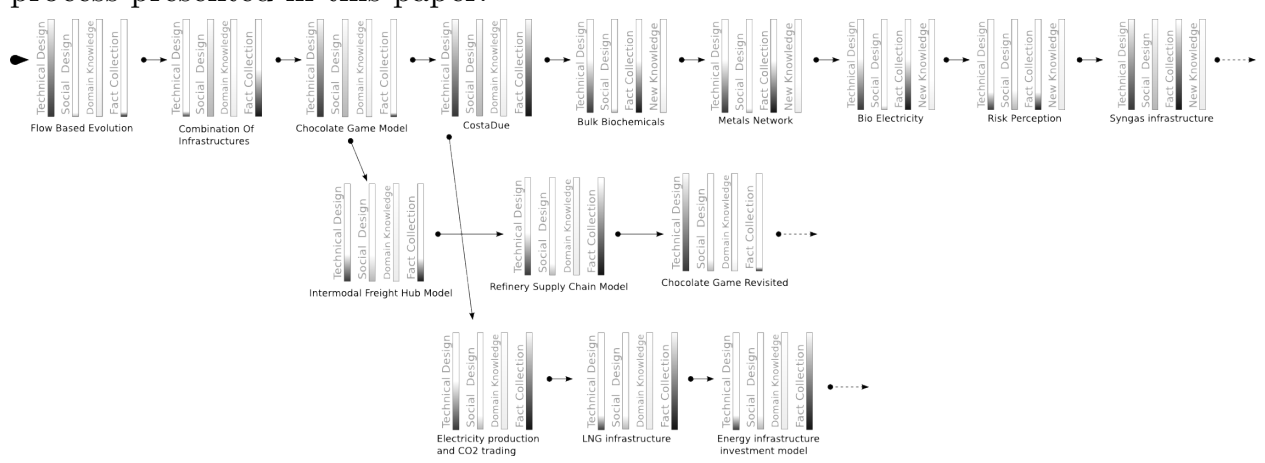

Figure 2 Evolutionary pathway of models

The foundation of the model is based on a physical and socio-economic model where agents must buy resources and then sell what they produce. From this simple concept, supply chains self-assemble. The case studies performed investigate different technological systems and different behavioral aspects. Several layers of 
behavior can be explored ranging from more immediate daily operational concerns, to longer term strategic decisions. The assumption underlying this foundation is that one needs to have an appropriate model for day-to-day operations before one can model strategic behavior successfully [Dijkema et al., 2007].

After three models used for testing (see figure 2), the first large scale model explored industrial co-evolution and examined the pathways by which an existing industrial region could transition into a bio-based cluster [Blokker, 2006, Nikolic and Dijkema, 2007]. This model served as an experimental platform for exploring more advanced programming techniques such as the use of an inference engine [Forgy, 1982] for decision-making. The three subsequent models examined case studies involving bio-based bulk chemical industries, copper and aluminum metals networks, and bio-electricity.

The bio-based bulk chemical case study explored how different combinations of supply chains would emerge given various economic conditions and decision-making typologies. This model examined the possibility of reversing the agent problem. Instead of asking what type of industrial-infrastructure network would evolve given the current economic conditions, we explored the questions that given a desired network, which environmental conditions would be able to support it. This gave the rise to the notion of "distance to reality" for a wanted network. This case additionally incorporated multi-criteria analysis as a way to model actor behavior that leads to system evolution [van Krevelen, 2007].

The copper and aluminum metals network case explored different frontiers by examining scenarios on demands of metals, economic growth, the efficiency of processes, and the substitution rate between both metals. Additionally, more sophisticated economics was employed, as investment decisions were made based on Net Present Value and Internal Rate of Return calculations. Agents also had the option to shut down their technologies temporarily while unfavorable economic conditions prevailed [Sakamornsnguan, 2007]. Elaborate economic development scenarios were introduced.

The case on bio-electricity focused on the integration of Life Cycle Analysis (LCA) [Guinee, 2002] within the Agent Based Modeling (ABM) framework. Since one of the motivators for bio-electricity is the potential for $\mathrm{CO}_{2}$ reductions, LCA is an important tool due to its ability to account for emissions occurring throughout global supply chains. The recent controversy on the conversion of rain forest into palm oil plantations [Fargione et al., 2008] has demonstrated the importance of such a global perspective. Combining LCA within ABM enables a type of dynamic LCA where the ABM is used to generate complexity due to the interactions of the agents, and the LCA is used as a type of network metric to analyze the characteristics of the system that has emerged [Davis, 2007, Davis et al., 2009].

Next to this model series, a series of power sector models were constructed [Chappin, 2006, Chappin and Dijkema, 2009, accepted]. In these models, investment in electricity generation technology is central, but also power and fuel trade is modeled and different carbon policies, such as emission-trading and carbon taxation have been implemented to assess their potential impact on the system. Power producing agents strategically manage by investing or disinvesting in power plants with unique sets of criteria to do so. In addition, electricity producing agents exhibit operational behavior by negotiation and engaging in contracts. The modeled system contains also other agents, i.e. government, markets for $\mathrm{CO}_{2}$ rights, 
power and fuels, power consumers and the underlying technology (power plants, consumer technology, physical networks and flows) [Chappin and Dijkema, 2009]. Scenario analysis was used operationalize the parameter space and outplay system developments under different exogenous conditions and trends.

An entirely different direction the base model has evolved is in the description of micro-processes inside households and firms. A micro combined heat and power model generation model of households has been constructed for the studying the dynamics of distributed power generation [van Dam et al., 2008]. Similarly, a detailed model of an oil refinery is being developed, modeling both the social and technical aspects of oil firms functioning. In the more infrastructure oriented series of models, work is in progress on a container transport hub model to examine the ideal location of a large scale transport hub given distance cost as well as noise and pollution disturbance for population living around it[Sirikijpanichkul et al., 2007].

\section{Results, Discussion and Conclusions}

This paper presented several different models, each model emerging from the previous one. They have provided a wealth of insights on $\lambda$-system evolution and are without a doubt useful. But what are the strengths and weaknesses, the limitations and the possibilities of those models?

\subsection{Models created}

The main thing that Agent Based Models of $\lambda$-system evolution can do is to help us explore and provide a sense of the range of possible system attractors, and give us a sense of possible futures.

By examining the models across a wide range of parameter values, a map or pattern of possible future development can be seen, depending on, for example, market changes, the introduction of new technologies, new policy introductions or changes in management styles.

The created models are built on objective physical characteristics and mechanisms of technical networks and on the beliefs and assumptions of stakeholders about the properties and mechanisms of the social network. Using the created ABMs of $\lambda$-system evolution provides us with a formal mechanism for testing the understanding and the intuition of the stakeholders. The ABMs often surprise us by showing emergent properties - logical but nonetheless surprising - caused by unexpected and unpredicted interactions of agents.

Compared to what the models can do, there are of course infinitely more things they cannot do. However, there are two main limitations relevant to understanding evolving $\lambda$-system evolution.

Because of the their focus, all models produced so far are unable to answer questions at the tactical or operational level. The models are not meant to aid the decision-making process on selecting suppliers, shutting down or starting a production of a single plant, etc. The models are meant to support strategic thinking only. This limitation, however, applies to the models developed. It is relatively straightforward to create the next generation of models that will are suitable to help us answer those kinds of questions. 
On a more fundamental level, there is a theoretical limit to what any generative model of an evolving system can do. As previously discussed, evolution is intractable. That means that there can never be a model that can exactly predict the future state of an evolving system. We might find models that provide good enough predictions, for varying levels of that qualification, given certain resources.

In order to conceptually map an agent-based model's properties, a short annotated Strengths, Weaknesses, Opportunities and Threats (SWOT) analysis is performed.

Strengths of Agent Based models are:

System representation They are multiformal, isomorphic and isodynamic with $\lambda$-systems. Unlike more traditional, top-down modelling tools, there is a good correspondence between the structure and dynamics of reality and the model.

Intuitive understanding From our experience in interacting with stakeholders, the users and modelers seem to intuitively understand the behavior of agents and their interaction, just as humans tend to think about entities having goals and 'wanting' things.

Weaknesses of Agent Based models are:

Data requirement As the required realism increases, the data requirement rises exponentially, quickly becoming impractical.

Implementation ABMs are still not straightforward to implement. The modelling tools used in this thesis, and the majority of tools reported in the literature, require computer programming skills for all but the simplest of agent descriptions.

Opportunities for the models are:

New paradigm ABMs are in general seen as something new and exciting. Modelers and users alike are drawn to shiny new toys.

Collective modelling Compared to more top-down models, the distributed nature of collective modelling makes it relatively easy to split the creation of a bottom-up model among different people.

Threats to the models' use and acceptance are:

Oversold modelling In the past, modelling (System Dynamics, Operations Research, etc.) has been actively promoted by modelers to policy makers with the promise of a rational prediction of future system states. Often, these predictions have turned out to be erroneous, raising doubts about the usefulness of modelling in general. Users have to be educated about the scope and relevance of Agent Based Model, and the modelers need to be more careful about their claims of prediction.

Over-dependency on models Once policy makers have accepted a model and are comfortable with its use, they tend to use it in ways not meant by modelers, leading to erroneous conclusions. This is harmful both to policy and model use.

Top-down tradition Because of the relative novelty of Agent Based Modeland the prevalence of the traditional top-down, command and control paradigm, users seem to not fully understand the bottom-up, distributed approach to modelling.

\subsection{Modelling Process}

As was done with the model outcomes, we now present a short, annotated SWOT of the co-evolutionary modelling process.

The strengths of the modelling process are: 
Socially accepted Since the modelling process involves a lot of stakeholder interaction, the process and its outcomes are likely to be accepted by the stakeholders.

Adaptive Since it is co-evolutionary, the process can adapt to changes in stakeholder preferences, new insights, etc.

Capacity building By focusing on a continuous improvement of the existing models, the process is suitable to capacity building and deepening of existing insights by research groups.

High throughput Once the process has been established, it becomes a proverbial paper machine, producing new outcomes very quickly and effectively.

The weaknesses of the modelling process are:

Expensive The co-evolutionary modelling process is expensive. If it is performed well, it involves many people and lots of time.

Slow takeoff The process requires a lot of up-front time and effort investment before yielding results. Creating the social network, understanding the real problem the stakeholder has, creating ontologies, setting up the simulation engine, etc. is time consuming.

Quality of social network The process is highly dependent on the quality of the social network and individual contributions by its members.

Far removed The process is relatively new and is often seen by stakeholders as being far removed from their daily reality, experimental and too complicated (high-end complex science). It takes time for them to become accustomed to the required way of working.

The main opportunities for the modelling process are:

Integrative The modelling process is explicitly meant to integrate different types of knowledge from different people into one unified whole.

Service-oriented The process creates continually improving insights over time, making it very suitable for a service-oriented, consulting-type business model for the research group.

The main threats to the modelling process are:

Path dependency/lock-in As it is evolutionary, the process is heavily dependent on its history. If the requirements of the stakeholders or the research topic of the group changes, a new investment must be made to restart the process.

Fragile The process is relatively fragile to changes in the composition and performance of the social network. If key individuals leave, or if the cooperative atmosphere is disturbed, the process can fail.

\subsection{Discussion}

The main insight gained from the modelling process is that scientific research is a highly chaotic evolutionary process during which we must make errors. This process is never completed, achieving at best 'good enough' results. This insight will be elaborated below.

The modelling process is explicitly co-evolutionary. Evolution is a process of making mistakes. When actively evolving a socio-technical design, either at the scale of models or at the scale of $\lambda$-systems, mistakes will inevitably be made. Such mistakes are useful, either as lessons in how not to do things or as unexpected improvements. Success or failure is arguably determined by a system's ability to gracefully deal with mistakes, whether through the ability to learn from them or 
through the ability to undo them. The modelling process requirements of modularity, versioning and authorship directly contribute to this aspect.

From a co-evolutionary perspective, recording all generated data/knowledge becomes imperative. We are bound to repeat the mistakes made earlier, and without a good historic record we would not be able to recognize them and rectify them in time.

Science, just as evolution, is never finished. Scientific results are never perfect, as the coupled fitness landscape of human understanding of the world we live it changes and deforms continually, raising the bar continuously. Instead of aiming for a grand goal by saying "it will be great once it is done", evolution teaches us that gradual progress and continuous improvement through local optimization is the way to go. Speaking in software terms, evolving processes are in a "perpetual beta" state, always incomplete but useful as is.

The notion of chaotic and never finished progress leads us to rethink the general approach to $\lambda$-system design. Instead of aiming for a perfectly optimal solution, we must realize that the most we can achieve is a good enough state, as both the problem formulation and the problem solution change all the time. The notion of good enough requires that we consider what would be a minimal acceptable operational threshold of a system's performance and allow the system to settle into any state that is above that. This way we allow the system's internal dynamics to run its course, and the unwanted side effects that occur when a system is far removed from its attractor are reduced.

\subsection{Conclusions}

Summarizing the above discussion, the co-evolutionary modelling process as presented in this paper can integrate many different formalisms into a single coherent vision on $\lambda$-system evolution. It can involve many different participants in collaborating on a greater whole, greatly increasing the overall output of the process. The process, being a Complex Adaptive Systems itself, can display emergent, surprising outcomes. If the process requirements are followed carefully, these emergent outcomes will be useful and insightful.

On the other side, the process cannot be used as a top-down steering mechanism, as it is by design bottom-up and distributed. Also, the co-evolutionary process can not guarantee any particular outcome, due to its chaotic nature, and finally, the co-evolutionary modelling process is not something one can perform alone.

When should the process be used? The process is suitable in supporting longterm multidisciplinary research with a coherent theme. It is useful when the modelers wish to build strong relationships with stakeholders, and the problem addressed is complex, multi-actor, multi-perspective and multi-scale.

The presented model should not be used to create 'quick and dirty', one off models, nor should it be used when it is important to urgently answer a new, burning question. The process is a long-term, multi-person effort that does not downscale well. It is also not suitable for rapid model prototyping and testing of ideas, unless they are evolutionary off-shoots of a larger modelling process. 


\section{Acknowledgments}

The authors would like to acknowledge the helpful suggestions of two anonymous reviewers and the Next Generation Infrastructures foundation for their financial support.

\section{References}

K. Antelman. Do open-access articles have a greater research impact. College $\mathcal{E}$ Research Libraries, 65(5):372-382, 2004.

W.R. Ashby. Variety, Constraint, and the Law of Requisite Variety. Modern Systems Research for the Behavioral Scientist. Hawthorne, NY: Adline de Gruyter, 1968.

Wiebe E. Bijker, Thomas Parke Hughes, and Trevor J. Pinch. The Social construction of technological systems : new directions in the sociology and history of technology. MIT Press. Cambridge, Mass, 1987.

Sjoerd Blokker. Coevolution in the process industry: An agent based model for the Eemshaven chemical cluster. Master's thesis, TU Delft, Faculty of Chemical Engineering, 2006.

E. J. L. Chappin. Carbon Dioxide Emission Trade Impact on Power Generation Portfolio, Agent-based Modelling to Elucidate Influences of Emission Trading on Investments in Dutch Electricity Generation. Delft University of Technology, Delft, 2006.

E. J. L. Chappin and G. P. J. Dijkema. On the impact of $\mathrm{CO}_{2}$ emission-trading on power generation emissions. Technological Forecasting 83 Social Change, 76(3): 358-370, 2009. doi: 10.1016/j.techfore.2008.08.004.

E. J. L. Chappin, G. P. J. Dijkema, and L. J. de Vries. Carbon policies: Do they deliver in the long run? In P. Sioshansi, editor, Carbon Constrained: Future of Electricity, Global Energy Policy and Economic Series. Elsevier, 2009. in press.

E.J.L. Chappin and G.P.J. Dijkema. Agent-based modeling of energy infrastructure transitions. International Journal of Critical Infrastructures, 2009, accepted.

Charles Darwin. The Origin of the Species. Penguin books, 1985.

C. Davis, I. Nikolic, and G.P.J. Dijkema. Integration of Life Cycle Assessment Into Agent-Based Modeling. Toward Informed Decisions on Evolving Infrastructure Systems. Journal of Industrial Ecology, 13(2):306-325, 2009.

C.B. Davis. Integration of life cycle analysis within agent based modeling using a case study on bio-electricity. Master's thesis, Delft University Of Technology, November 2007.

Richard Dawkins. The Selfish Gene. Oxfrd University press, 1990. 
DA DeLaurentis and WA Crossley. A Taxonomy-based Perspective for Systems of Systems Design Methods. Systems, Man and Cybernetics, 2005 IEEE International Conference on, 1, 2005.

D.C. Dennet. Darwin's Dangerous Idea: Evolution and the Meanings of Life. Simon \& Schuster, New York, 1996.

G.P.J. Dijkema, I. Nikolic, S. Biesheuvel, S. IJsselstijn, J. Baggen, J. Stoop, O. van Riet, H.A. Weustenenk, and J. Smits. More infrastructure capacity per acre? methoology to establish the (im)possibilities of combination of infrastructures. In Toh Ah Cheong and Chan Eng Soon, editors, Proceedings of the 2nd International Maritime-Port Technology and Development Conference Sept. 26-28 200\%, number ISBN: 978-981-05-9102-1, pages 127-132. Singapore: Research Publishing Services, 2007.

T.M. Egyedi and Z. Verwater-Lukszo. Which standards' characteristics increase system flexibility? Comparing ICT and batch processing infrastructures. Technology in Society, 27(3):347-362, 2005.

J.M. Epstein. Agent-based computational models and generative social science. Complexity, 4(5):41-60, 1999.

J. Fargione, J. Hill, D. Tilman, S. Polasky, and P. Hawthorne. Land Clearing and the Biofuel Carbon Debt. Science, 319(5867):1235, 2008.

C. L. Forgy. Rete a fast algorithm for the many pattern many object pattern match problem. Artificial Intelligence, 19(1):17 - 37, 1982. ISSN 0004-3702.

J. Gall. The Systems Bible: The Beginner's Guide to Systems Large and Small: Being the Third Edition of Systemantics. General Systemantics Press, 2002.

J. B. Guinee. Handbook on Life Cycle Assessment: Operational Guide to the ISO Standards. Kluwer Academic Publishers, 2002.

PM Herder. Process Design in a Changing Environment: Identification of Quality Demands Governing the Design Process. Delft University Press, Delft, The Netherlands, 1999.

W. Hordijk and S. A. Kauffman. Correlation analysis of coupled fitness landscapes. 10(6):41 - 49, 2005. ISSN 1076-2787.

T. Karp. Transforming organisations for organic growth: The DNA of change leadership. Journal of Change Management, 6(1):3-20, 2006.

S. A. Kauffman and S. Johnsen. Coevolution to the edge of chaos - coupled fitness landscapes, poised states, and coevolutionary avalanches. J. Theoretical Biol., 149(4):467-505, 1991.

T.B. Kepler, M.A. Marti-Renom, S.M. Maurer, A.K. Rai, G. Taylor, and M.H. Todd. Open Source Researchthe Power of Us. Australian Journal of Chemistry, $59(5), 2006$.

D.C. Mikulecky. The emergence of complexity: science coming of age or science growing old? Computers and Chemistry, 25(4):341-348, 2001. 
I. Nikolic and G.P.J. Dijkema. Framework for Understanding and Shaping Systems of Systems The case of industry and infrastructure development in seaport regions. System of Systems Engineering, 200\%. SoSE'0\%. IEEE International Conference on, pages 1-6, 2007.

Igor Nikolic. Co-Evolutionary Process For Modelling Large Scale Socio-Technical Systems Evolution. PhD thesis, Delft University of Technology, 2009.

Igor Nikolic, Emile J. L. Chappin, Chris Davis, and Gerard P. J. Dijkema. On the development of agent-based models for infrastructure evolution. In International Conference on Infrastructure Systems - Building Networks for a Brighter Future, Rotterdam, The Netherlands, November 2008. NGInfra Foundation.

S.E. Page. The Difference: How the Power of Diversity Creates Better Groups, Firms, Schools, and Societies. Princeton University Press Princeton, NJ, USA, 2007.

K.R. Popper. The Logic of Scientific Discovery. Routledge, 2002.

K. Sakamornsnguan. Supporting condition for sustainable metal network development: Agent-based simulation on infrastructural investment of cu and al. Master's thesis, Delft University of Technology, October 2007.

A. Sirikijpanichkul, K.H. van Dam, L. Ferreira, and Z. Lukszo. Optimizing the Location of Intermodal Freight Hubs: An Overview of Agent Based Modelling Approach. J Transp Sys Eng \& IT, 4, 2007.

K.H van Dam, M. Houwing, Z. Lukszo, and I. Bouwmans. Agent-based control of distributed electricity generation with micro combined heat and power - Crosssectoral learning for process and infrastructure engineers. Computers and Chemical Engineering, 32(1-2):205-217, 2008.

R. E. J. van Krevelen. Two analysis methods for the evaluation of cluster formation. assessment using a case study on biofuel and bulk chemicals clusters in the netherlands. Master's thesis, Delft University of Technology, September 2007.

M.M. Waldorp. Complexity: The emerging science at the edge of order and chaos. Simon and Schuster, New York, 1992.

A. W. Westerberg, E. Subrahmanian, Y. Reich, S. Konda, D. P. Cunningham, A. H. Dutoit, H. L. Granger, S. Konda, K. C. Marshall, R. C. Milliken, I. A. Monarch, J. P. Neergaard, R. H. Patrick, and M. E. Thomas. Designing the process design process. Computers \& Chem. Engineering, 21:S1-S9, 1997. 